\title{
PHÂN TÍCH ĐẶC TÍNH KHÍ ĐộNG LỰC XE TIẾT KIỆM NHIÊN LIỆU THAM GIA CUỘC THI HONDA EMC
}

\author{
ĐẬNG TIÊN PHÚC \\ Khoa Công nghệ Động lực, Truờng Đại học Công nghiệp Thành phố Hồ Chí Minh \\ dangtienphuc@iuh.edu.vn
}

Tóm tắt. Bài báo này trình bày nghiên cứu đặc tính khí động lực học xe tiết kiệm nhiên liệu tham gia cuộc thi Honda EMC thông qua phân tích mô hình xe ba bánh. Tác giả sử dụng phương trình RANS kết hợp mô hình rối Realizable $k-\varepsilon$ trong phần mềm thương mại Ansys Fluent để mô phỏng đặc tính khí động lực học mô hình xe ba bánh. Bài báo trình bày phương pháp và kết quả mô phỏng sự phân bố áp suất, sự phân bố vận tốc quanh mô hình. Kết quả nghiên cứu được thể hiện thông qua các hình ảnh và giá trị mô phỏng tính toán $\mathrm{C}_{\mathrm{d}}$ đồng thời giúp hiểu rõ hơn đặc tính khí động lực học quanh mô hình xe ba bánh tham gia cuộc thi Honda EMC.

Từ khóa: Mô phỏng CFD, khí động lực học ô tô, Honda EMC, hệ số cản, RANS.

\section{AERODYNAMIC STUDY OF HONDA ECO MILEAGE CHALLENGE CAR}

\begin{abstract}
This paper studies the aerodynamic characteristics of Honda Eco Mileage Challenge car. Numerical investigations were conducted with the motor tricycle model. The results of the numerical simulations were obtained using Reynolds-averaged Navier-Stokes (RANS) equations with Realizable $k-\varepsilon$ model by commercial software ANSYS. The Time-averaged velocity field, magnitude of the velocity, static pressure magnitude of the flow fields around model are presented in this paper. The results of this study elucidate the key aerodynamic characteristics around motor tricycle model. The results obtained from the study are presented graphically and values of drag coefficient $\left(\mathrm{C}_{\mathrm{d}}\right)$ were calculated and validated, which helps better understanding aerodynamic characteristics around motor tricycle model.
\end{abstract}

Keyword: CFD simulation, Vehicle aerodynamics, Honda EMC car, drag coefficient, RANS.

\section{GIÓI THIÊUU}

Khí động lực học ô tô đã phát triển trong nhiều thập kỷ qua và đã trở thành một yếu tố quan trọng đối với các dòng xe hơi nên rất nhiều các công trình khoa học trong lĩnh vực này được công bố [1-4]. Khi ô tô chuyển động trong môi trường không khí sẽ bị các lực và mô ment khí động học tác dụng làm ảnh hưởng đến tính năng chuyển động của ô tô và lượng tiêu hao nhiên liệu. Tổng trọng lượng và hình dạng bên ngoài xe là một trong những yếu tố quan trọng nhằm cải tiến đặc tính khí động lực học của xe [5-7]. Để hiểu được sự tác động khí động lực học lên ô tô, các nhà nghiên cứu đã tiến hành nghiên cứu đặc tính khí động lực học quanh ô tô bằng hai phương pháp mô phỏng và thực nghiệm. Một số nghiên cứu mô phỏng về ảnh hưởng khí động lực học đến ô tô chẳng hạn như tác giả [8] đã nghiên cứu về hệ số cản xe buýt khi thay đổi thiết kế hình dạng xe buýt nhằm làm giảm tiêu hao nhiên liệu và khí xả gây ô nhiễm môi trường. Kết quả, tác giả đã kết luận rằng với mô hình mô phỏng rối Realizable $k-\varepsilon$, khi thay đổi hình dạng xe buýt so với hình dạng ban đầu, hệ số cản giảm $28 \%$ và tiết kiệm được $20 \%$ nhiên liệu khi xe di chuyển với vận tốc 80 $\mathrm{km} / \mathrm{h}$, tác giả [9] đã nghiên cứu lực cản khí động lực học của xe du lịch và kết luận rằng kết cấu vùng đầu xe, đuôi xe có ảnh hưởng lớn đến lực cản khí động tác dụng lên vỏ xe. Bên cạnh đó, nhiều tác giả thực hiện các thí nghiệm thực tế, cụ thể tác giả [10] đã thực hiện nghiên cứu thực nghiệm PIV phân tích sự ảnh hưởng của góc nghiêng đuôi đến đặc tính dòng khí quanh mô hình Ahmed. Kết quả, tác giả đã trình bày các hình ảnh cấu trúc dòng khí ở phần đuôi mô hình tại mặt phẳng đối xứng của mô hình Ahmed. Đồng thời tác giả cũng mô tả các điểm tập trung và các điểm yên ngựa trong dòng khí ở sau đuôi mô hình. Tác giả [11] đã tiến hành thực nghiệm để phân tích ứng xử khí động lực học của bộ tạo xoáy tương ứng với bốn vị trí khác nhau trên ô tô. Kết quả, tác giả đã trình bày các hệ số cản và hệ số nâng giảm nhiều nhất là $4.53 \%$ và $2.55 \%$ trong trường hợp bộ tạo xoáy có cạnh tiếp xúc của hai chi tiết nằm phía sau xe và một chi tiết nằm giữa đuôi ô tô. Tác giả [12] đã thực hiện thử nghiệm khí động lực học trên mô hình Mira với kết quả đạt được là làm rõ cấu trúc dòng rối sau mô hình và phát hiện cơ chế hình thành dòng khí tức thời trên mô hình. 
Cuộc thi Lái xe sinh thái - Tiết kiệm nhiên liệu Honda EMC là sân chơi nơi những người tham gia áp dụng những ý tưởng và công nghệ của mình vào động cơ 4 thì của Honda để thiết kế xe tiết kiệm tiêu hao nhiên liệu, di chuyển quãng đường xa nhất với llít nhiên liệu [13]. Bên cạnh việc nghiên cứu cải tiến hiệu suất của động cơ, áp dụng các công nghệ mới, các đội tham gia còn thiết kế cải tiến hình dạng của xe nhằm cải thiện lực khí động học tác dụng lên xe. Thực tế, trọng lượng của xe là một trong những yếu tố chính mà các đội luôn nghiên cứu để giảm bằng cách sử dụng loại vật liệu mới có kết cấu cứng vững và trọng lượng nhẹ. Ngoài ra, hình dạng của xe cũng là một trong những nguyên nhân ảnh hưởng đến khả năng chuyển động của xe. Trong khi tốc độ xe tăng lên, lực cản lăn tương đối không thay đổi nhưng lực cản khí động tăng theo bình phương vận tốc. Khi tốc độ xe lớn hơn $35 \mathrm{~km} / \mathrm{h}$, lực cản khí động lúc này lớn hơn lực cản lăn của xe [14]. Trong bài báo này, tác giả tiến hành nghiên cứu đặc tính khí động lực học xe được thiết kế tham gia cuộc thi Honda EMC nhằm cung cấp cho người đọc cái nhìn tổng quan và rõ nét hơn sự ảnh hưởng của khí động lực học. Đây cũng chính là một bước quan trọng trong quá trình thiết kế hình dạng xe. Để đạt được mục tiêu này, tác giả sử dụng phương trình Reynolds trung bình hóa cho dòng chảy rối (RANS) kết hợp với mô hình rối Realizable $k-\varepsilon$ để mô phỏng đặc tính khí động lực học dòng khí.

\section{MÔ HÌNH XE THAM GIA CUỘC THI HONDA EMC VÀ MIỀN TÍNH TOÁN}

\subsection{Xây dựng mô hình 3D}

Dựa và quy định kích thước xe tham gia cuộc thi của ban tổ chức Honda, mô hình xe được thiết kế với hình dạng các thông số được mô tả cụ thể trên hình 1 và hình 2 .

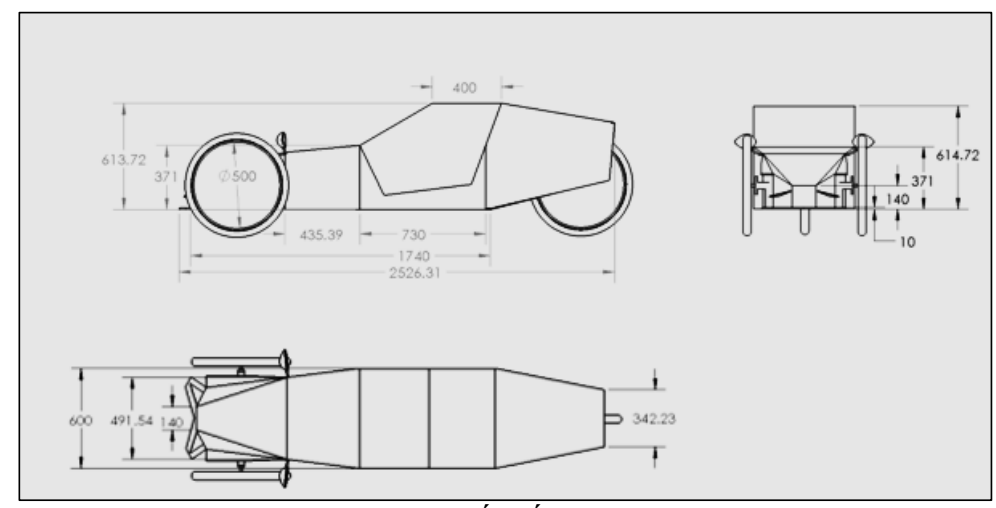

Hình 1: Kích thước thiết kế xe theo qui định Honda EMC.

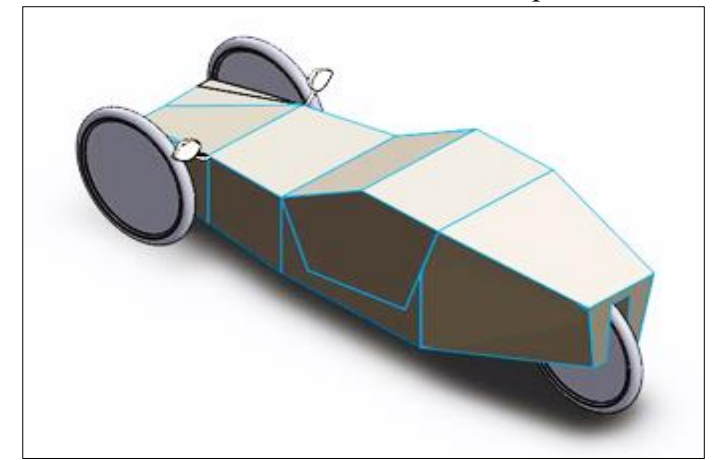

Hình 2: Mô hình 3D xe tham dự cuộc thi Honda EMC.

Để hiểu rõ đặc tính khí động lực học trên mô hình nghiên cứu, trong quá trình mô phỏng phân tích sẽ thể hiện sự phân bố áp suất, vận tốc tại mặt phẳng $(\mathrm{P} 1)$ và mặt phẳng $(\mathrm{P} 2)$ mô tả ở hình 3 . Trong đó, mặt phẳng $\mathrm{P} 1)$ là mặt phẳng cắt dọc đối xứng mô hình, mặt phẳng $(\mathrm{P} 2)$ là mặt phẳng cắt ngang và và song song với mặt đường. 


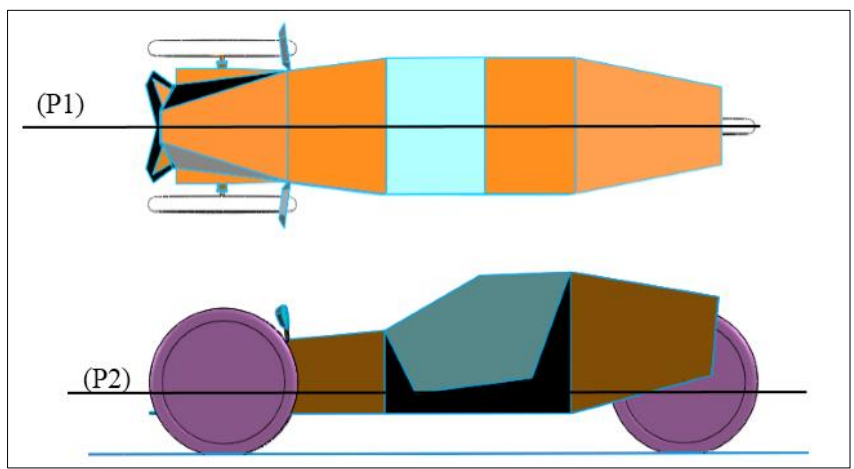

\subsection{Miền tính toán}

Hình 3: Mặt phẳng thể hiện sự phân bố áp suất, vận tốc quanh mô hình.

Miền tính toán là vùng không gian bao quanh vật thể được giới hạn trong quá trình mô phỏng. Miền tính toán phải có kích thước đủ lớn được giới hạn bởi các mặt phẳng để đảm bảo dòng không khí không chịu ảnh hưởng của mô hình nghiên cứu. Tuy nhiên cũng không thể lựa chọn miền tính toán quá lớn dẫn đến làm lãng phí tài nguyên máy tính, tăng thời gian mô phỏng tính toán. Vì vậy, kết hợp với các công trình nghiên cứu của các tác giả $[4,15,16]$, tác giả tiển hành xây dựng miền tính toán với các thông số được mô tả ở hình 4 với áp suất khí trời, vận tốc $15 \mathrm{~km} / \mathrm{h}$ và chiều dài xe $\mathrm{L}=2525 \mathrm{~mm}$.

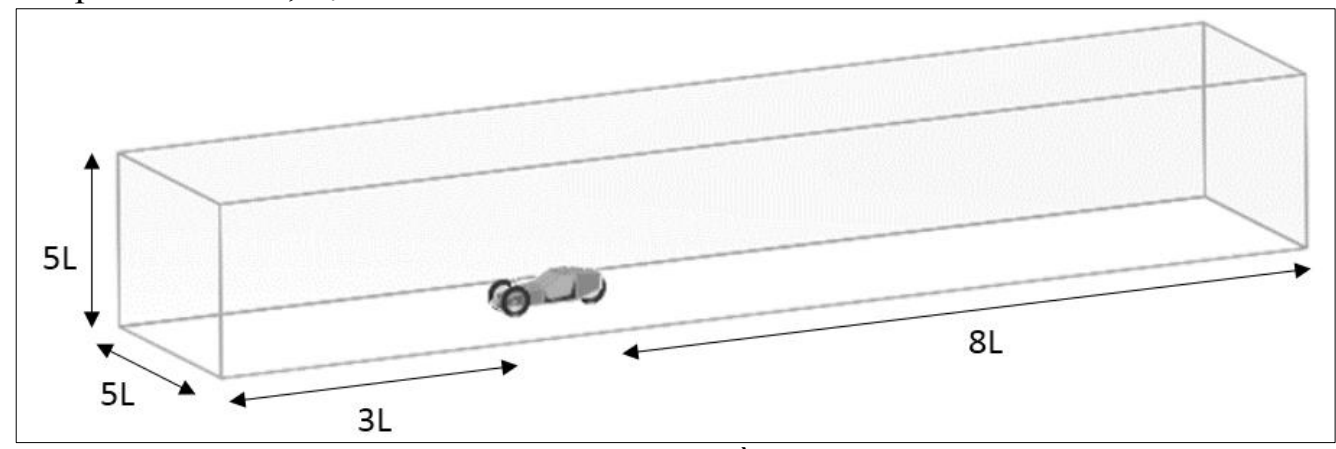

Hình 4: Kích thước của miền tính toán.

\section{PHƯƠNG PHÁP MÔ PHỎNG SỐ}

\subsection{Phương trình điều chỉnh}

Để mô tả chuyển động của lưu chất thường sử dụng phương trình Navier-Stokes là hệ các phương trình bảo toàn khối lượng (phương trình liên tục), phương trình bảo toàn động lượng và phương trình bảo toàn năng lượng. Trong nghiên cứu về khí động lực học ô tô đặt giả thiết rằng chất khí không chịu nén, do đó, bài toán khí động lực học chỉ còn lại hai phương trình là phương trình liên tục và phương trình bảo toàn động lượng.

Phương trình liên tục:

$$
\frac{\partial u}{\partial x}+\frac{\partial v}{\partial y}+\frac{\partial w}{\partial z}=0
$$

Phương trình bảo toàn động lượng:

$$
\partial_{t} u_{i}+u_{j} \partial_{j} u_{i}=-\frac{1}{\rho} \partial_{i} p+v \partial_{j} \partial_{j} u_{i}
$$

Trong bài nghiên cứu này, tác giả sử dụng phương trình Reynolds Navier - Stokes trung bình hóa (RANS) để giải bài toán khí động học:

$$
\begin{aligned}
& \partial_{i} \bar{u}_{i}=0 \\
& \partial_{t} \bar{u}_{i}+\bar{u}_{j} \partial_{j} \bar{u}_{i}=-\frac{1}{\rho} \partial_{i} \bar{p}+\frac{1}{\rho} \partial_{j}\left(\tau_{\mathrm{ij}}-\rho \overline{u_{i}^{\prime} u_{i}^{\prime}}\right)
\end{aligned}
$$

Ngoài ra, để giải phương trình RANS tác giả sử dụng mô hình rối Realizable $k-\varepsilon$, đây là mô hình rối đã được nhiều tác giả sử dụng trong nghiên cứu khí động lực học ô tô. 


\subsection{Chia lưới và điều kiện biên.}

Chia lưới chính là rời rạc hóa vùng không gian mô phỏng thành các phần tử để thực hiện tính toán gần đúng bằng phương pháp số. Số lượng phần tử của mô hình ảnh hưởng đến độ chính xác kết quả cũng như thời gian tính toán. Trong bài nghiên cứu tác giả sử dụng lưới tứ diện do mô hình mô phỏng phức tạp. Miền tính toán được chia lại với kích thước của phần tử lưới ở vùng biên và gần mô hình phân tích phải đủ nhỏ để đảm bảo độ chính xác của kết quả khi mô phỏng tính toán $[3,17]$, trong khi các vùng xa vùng biên và mô hình có kích thước của phần tử lưới lớn hơn nhằm tiết kiệm tài nguyên của máy tính cũng như thời gian tính toán. Mô hình xe tham dự cuộc thi Honda EMC đã chia lưới hoàn chỉnh được mô tả ở hình 5 với tổng phần tử lưới 942242 với kích thước lưới nhỏ nhất của phần tử lưới gần bề mặt là $2 \mathrm{~mm}$.

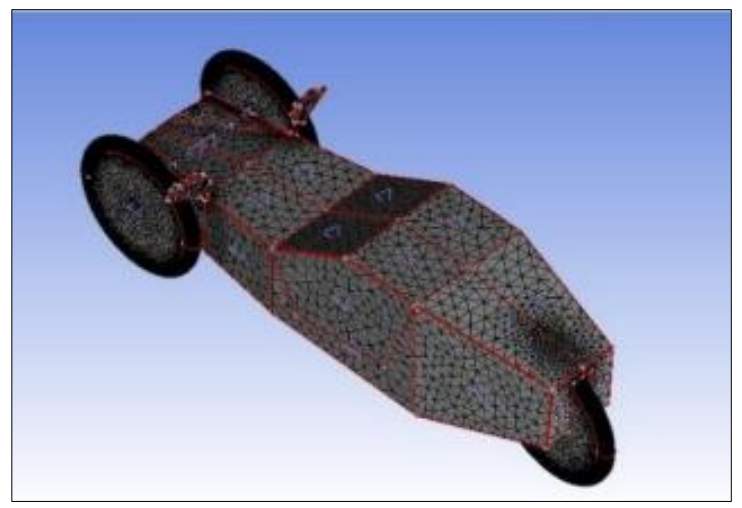

Hình 5: Mô hình xe tham dự Honda EMC đã chia lưới với dạng tứ diện không có cấu trúc

\section{KẾT QUẢ}

\subsection{Phân bố trường áp suất quanh mô hình.}

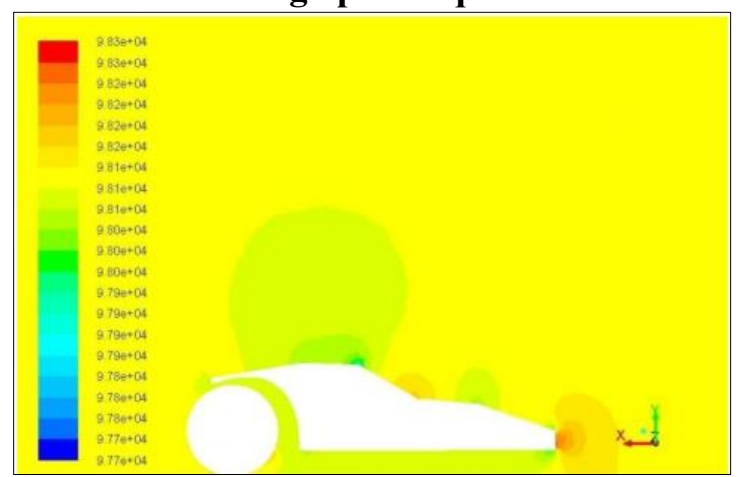

(a) Phân bố áp suất tại mặt phẳng $(\mathrm{P} 1)$

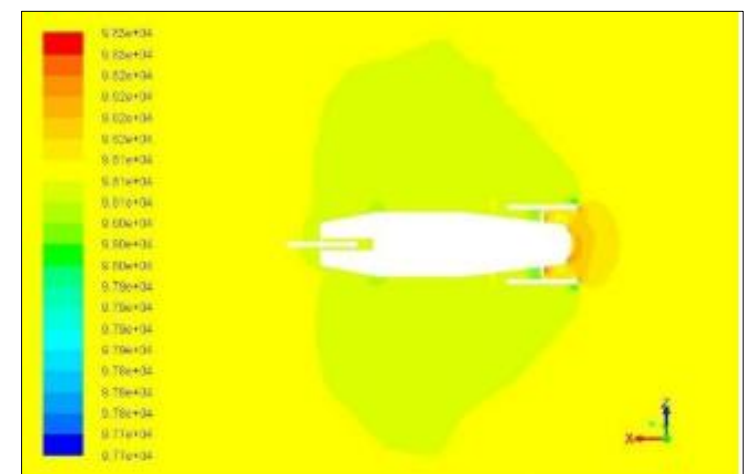

(b) Phân bố áp suất tại mặt phẳng $(\mathrm{P} 2)$

Hình 6: Phân bố áp suất tại mặt phẳng $(\mathrm{P} 1),(\mathrm{P} 2)$.

Quan sát trên hình 6 , dựa vào thang màu sắc được phân bố trên các mặt phẳng $(\mathrm{P} 1),(\mathrm{P} 2)$ và $(\mathrm{P} 3)$ xung quanh mô hình từ xanh da trời đến màu đỏ thể hiện độ lớn áp suất tại những vùng khác nhau quanh mô hình. Hình $5(\mathrm{a})$ thể hiện phân bố áp suất tại khu vực trước xe và vùng tiếp nối giữa nắp capo và kính chắn gió xung quanh mô hình nghiên cứu là lớn nhất. Bên cạnh đó, áp suất tại các khu vực thay đổi biên dạng như mũi xe, nắp capo và mui xe giảm nhiều nhất. Các hiện tượng này xảy ra do dòng khí khi tác động lên bề mặt mô hình làm xuất hiện các điểm tách rời, điểm ngưng tụ và các vùng xoáy xung quanh mô hình. Tại khu vực trước mô hình luôn tiếp xúc trực tiếp với dòng khí nên luôn có áp suất lớn hơn so với những vùng khác trên mô hình. Cụ thể, áp suất tại khu vực trước mô hình lớn hơn nhiều so với áp suất tại khu vực đuôi mô hình. Hình $5(\mathrm{~b})$ mô tả chi tiết khu vực trước xe xuất hiện vùng áp suất lớn là vị trí mũi xe, phía trước bánh xe và trục bánh xe. Chính sự chênh lệch áp suất ở các khu vực khác nhau xung quanh mô hình là yếu tố cơ bản tạo nên lực cản khí động khi ô tô di chuyển. Sự chênh áp giữa khu vực trước mô hình và khu vực đuôi mô hình càng lớn làm cho lực cản khí động càng tăng. Như vậy, để làm giảm lực cản khí động cần giảm sự chênh áp giữa khu vực phía trước và sau mô hình. 


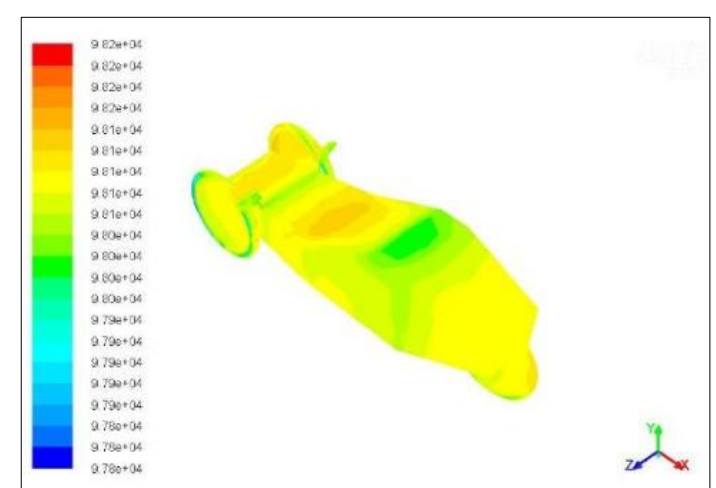

Hình 7: Phân bố áp suất trên mô hình xe tham dự Honda EMC.

Quan sát hình 7 thể hiện sự phân bố áp suất trên mô hình xe tham dự Honda EMC khi xe di chuyển với tốc độ $15 \mathrm{~m} / \mathrm{s}$. Tại khu vực đầu xe, phần trước của hai bánh xe trước và khu vực tiếp nối giữa nắp capo với kính chắn gió xuất hiện vùng phổ màu đậm hơn so với những vùng khác trên mô hình do sự tách rời của dòng khí, chính sự chênh áp này là một trong những nguyên nhân tạo nên lực cản khí động. Sự chênh áp càng lớn thì lực cản khí động càng tăng và để làm giảm lực cản khí động thì cần tăng áp suất phía sau mô hình.

\subsection{Phân bố trường vận tốc và phân bố dòng khí quanh mô hình}

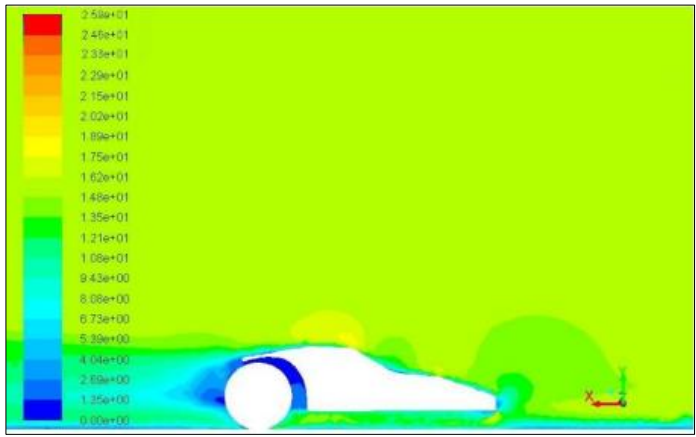

(a) Phân bố vận tốc tại mặt phẳng (P1)

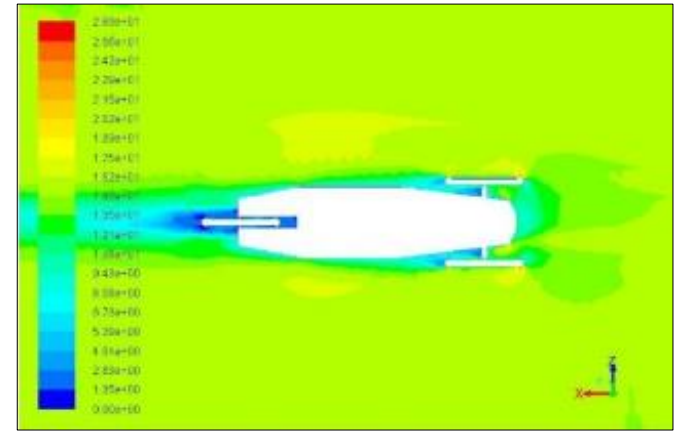

(b) Phân bố vận tốc tại mặt phẳng $(\mathrm{P} 2)$

Hình 8: Phân bố vận tốc tại mặt phẳng dọc $(\mathrm{P} 1)$ dối xứng quanh mô hình

Hình 8 thể hiện trường vận tốc tại mặt phẳng $(\mathrm{P} 1)$ và $(\mathrm{P} 2)$ quanh mô hình. Quan sát thấy dòng khí bị cản lại tại khu vực trước mô hình thang màu sắc từ xanh da trời đến màu đỏ thể hiện độ lớn vận tốc tại những vùng khác nhau quanh mô hình. Tại vùng đầu mô hình luôn luôn có vận tốc nhỏ do dòng khí khi di chuyển đến vùng này sẽ xuất hiện một điểm dừng. Ngay tại điểm dừng, vận tốc dòng khí bằng không và dòng khí sẽ chia làm hai phần, một phần đi dọc theo phía trên thân mô hình và một phần đi dọc theo phía dưới thân mô hình. Bên cậnh đó, biên dạng mô hình thay đổi gồm các góc bo tròn, góc nghiêng mà cụ thể là khu vực tiếp nối giữa nắp capo với kính chắn gió dẫn đến sự phân bố vận tốc tại những khu vực đó cũng thay đổi. Quan sát khu vực mui xe xuất hiện vùng màu đậm chứng tỏ tại khu vực này vận tốc dòng khí tăng lên đáng kể do sự thay đổi biên dạng mô hình đột ngột. Tại những khu vực có biên dạng thay đổi đột ngột, dòng khí bị tách rời đây là một trong những nguyên nhân làm thay đổi sự phân bố vận tốc quanh mô hình.

Quan sát hình 6 và hình 8 , mối liên hệ giữa phân bố áp suất và phân bố vận tốc tại các khu vực tiếp xúc với dòng khí xung quanh mô hình. Sự phân bố áp suất và vận tốc quanh mô hình luôn tuân theo định luật Bernoulli với khu vực áp suất cao chính là khu vực có vận tốc thấp.

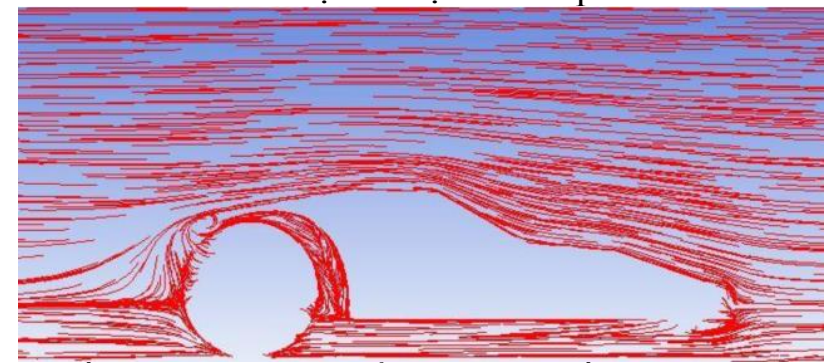

Hình 9: Phân bố dòng khí tại mặt phẳng dọc (P1) đối xứng quanh mô hình 
Hình 9 mô tả các đường dòng không khí chuyển động trên mặt phẳng dọc P1 quanh mô hình xe. Dòng khí chuyển động tương đối ổn định tại vùng đầu xe và không xuất hiện các vùng xoáy. Tuy nhiên, càng về phía đuôi xe dòng khí lại xuất hiện vùng xoáy tạo nên sự chênh lệch về áp suất giữa phía trước và sau xe, đây là một trong những nguyên nhân chính dẫn tới sự hình thành lực cản khí động lực học [1].

\subsection{Lụ̣c khí động lực học}

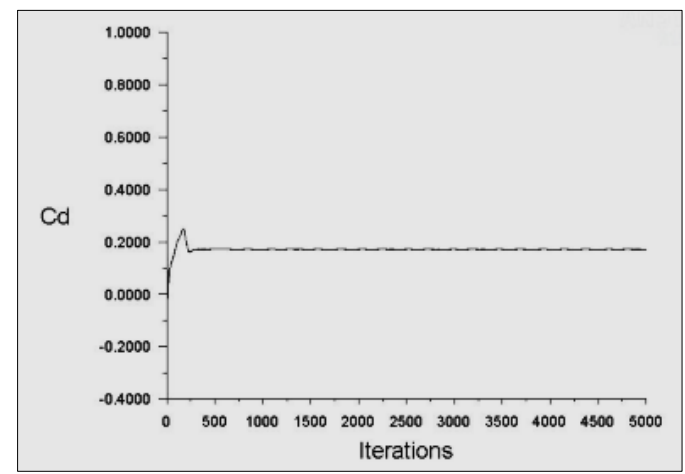

Hình 10: Biểu đồ thể hiện hệ số cản lăn $\mathrm{C}_{\mathrm{d}}$ sau tính toán mô phỏng

Kết quả tính toán mô phỏng trong nghiên cứu hệ số cản không khí với khoảng 5000 lần lặp thu được giá trị $\mathrm{C}_{\mathrm{d}}$. Dựa vào biểu đồ hình 10 , sau lần lặp thứ 500 hệ số $\mathrm{C}_{\mathrm{d}}$ có giá trị dần ổn định và kết quả mô phỏng $\mathrm{C}_{\mathrm{d}}=$ 0.17. So sánh với hệ số cản của tác giả [18] thực hiện nghiên cứu mô hình xe ba bánh hình dạng giọt nước có $\mathrm{C}_{\mathrm{d}}=0.15$ có sự sai lệch $14 \%$ giữa hai mô hình. Vậy để mô hình nghiên cứu đạt giá trị hệ số cản nhỏ hơn cần tiến hành cải tiến hình dạng mô hình tuy nhiên cần quan tâm đến các quy định của cuộc thi Honda EMC. Từ giá trị $\mathrm{C}_{\mathrm{d}}$ tiến hành tính được các lực cản thông qua công thức:

Lực cản không khí: $\quad F_{d}=\frac{1}{2} C_{d} A \rho U_{\infty}^{2}$

\section{KẾT LUẬN}

Bài báo đã trình bày kết quả tính toán mô phỏng đặc tính khí động lực học cho xe tiết kiệm nhiên liệu tham gia cuộc thi Honda EMC, sử dụng phương trình RANS kết hợp với mô hình rối Realizable $k-\varepsilon$ đối với mô phỏng CFD. Kết quả nghiên cứu thể hiện tại các khu vực có sự thay đổi đột ngột về biên dạng cụ thể khu vực đầu xe, khu vực tiếp giáp giữa nắp capo và kính chắn gió và khu vực đuôi xe có sự thay đổi lớn về sự phân bố áp suất cũng như vận tốc. Sự chênh lệch áp suất ở những khu vực này một trong những nguyên nhân ảnh hưởng lớn đến lực cản khí động tác dụng lên xe. Bên cạnh đó, bài báo này cũng đã minh họa rõ bằng hình ảnh sự phân bố áp suất, vận tốc quanh mô hình trên mặt phẳng cắt dọc đối xứng mô hình và mặt phẳng cắt ngang và và song song với mặt đường. Kết quả nghiên cứu là tiền đề để thực hiện tính toán mô phỏng trên mô hình ô tô thực tế, thông qua mô phỏng số CFD sẽ giải quyết được vấn đề thí nghiệm khí động lực học ô tô khi hiện nay phòng thí nghiệm khí động lực học ô tô ở nước ta còn rất hạn chế.

\section{CÁC KÝ HIỆU}

- u, v,w: Vận tốc của phần tử lưu chất theo 3 trục tọa độ $\mathrm{x}, \mathrm{y}, \mathrm{z}$.

- $\partial_{t} u_{i}$ : Đạo hàm riêng theo thời gian của $\mathrm{u}$.

- $\rho \quad$ : Khối lượng riêng của không khí $\left(\mathrm{kg} / \mathrm{m}^{3}\right)$.

- $\mathrm{p} \quad$ : Áp suất $\left(\mathrm{N} / \mathrm{m}^{2}\right)$.

- $\quad v \quad$ : Độ nhớt động học của không khí $\left(\mathrm{kg} \cdot \mathrm{m} / \mathrm{s}^{-1}\right)$.

- $\mathrm{C}_{\mathrm{d}} \quad$ : Hệ số cản.

- $\rho \overline{u_{i}^{\prime} u_{i}^{\prime}}$ : Úng suất Reynolds.

- $\tau_{\mathrm{ij}} \quad$ : Ten sơ ứng suất

- A $\quad$ : Diện tích cản chính diện $\left(\mathrm{m}^{2}\right)$

- $U_{\infty} \quad$ : Vận tốc chuyển động $(\mathrm{m} / \mathrm{s})$ 


\section{TÀI LIỆ THAM KHẢO}

[1] Hucho, W.H., Aerodynamics of Road Vehicles, Butterworth and Co. Publishing, Boston, MA, 1990.

[2] Katz, J., Race Car Aerodynamics: Designing for Speed, Bentley Publishers, Philadelphia, PA, 1995.

[3] Halil, S.H., Rami, S.E., Murat, A. and Ibrahim, D, Effects of rear spoilers on ground vehicle aerodynamic drag", International Journal of Numerical Methods for Heat \& Fluid Flow, 2014, 24(3): 627-642.

[4] Tien Phuc Dang, Zhengqi Gu, Zhen Chen, Numerical simulation of flow field around the race car in case, International Journal of Numerical Methods for Heat \& Fluid Flow, 2015, 25(8): 896- 1911.

[5] Heft, A., Indinger, T., and Adams, N., Introduction of a New Realistic Generic Car Model for Aerodynamic Investigations, SAE Technical Paper 2012, doi:10.4271/2012-01-0168.

[6] M. Desai, S. A. Channiwala and H. J. Nagarsheth, A Comparative assessment of two experimental methods for aerodynamics performance evaluation of car, Journal of scientific and industrial Research, 2008, 67: 518-522.

[7] C. Rajsinh B. and T. K. Raj R., Numerical investigation of external flow around the ahmed reference body using computational fluid dynamics, Research Journal of Recent Sciences, 2012, 1(9): 1-5.

[8] Siddhesh Kanekar, Prashant Thakre and E Rajkumar, Aerodynamic study of state transport bus using computational fluid dynamics. IOP Conference Series: Materials Science and Engineering, 2017, 263. 062052

[9] Đỗ Tiến Quyết, Nghiên cứu lực cản khí động học của xe du lịch bằng phần mềm Ansys, Tạp chí Nghiên cúu khoa học - Đại học Sao Đỏ, 2019, 1(64).

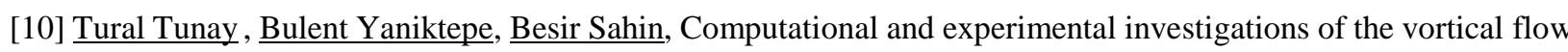
structures in the near wake region downstream of the Ahmed vehicle model, Journal of Wind Engineering and Industrial Aerodynamics, 2016, 159:48-64.

[11] G. Shankar and G. Devaradjane, Experimental and Computational Analysis on Aerodynamic Behavior of a Car Model with Vortex Generators at Different Yaw Angles, Journal of Applied Fluid Mechanics, 2018, 11(1), 285-295, 2018.

[12] Aerodynamic Characteristics of Mira Fastback Model in Experiment and CFD, International Journal of Automotive Technology, 2019, 20(4), 723-737.

[13] Http://bit.ly/Dangky-EMC-Honda-2020

[14] Barnard, R.H., Road vehicle aerodynamic design-an introduction, Mechaero Publishing, 2001, 2nd Revised Edition.

[15] M. Corallo , J. Sheridan, M.C. Thompson, Effect of aspect ratio on the near-wake flow structureof an Ahmed body, Journal of Wind Engineering and Industrial Aerodynamics, 2015, 147,95-103.

[16] Tural Tunay, Besir Sahin, Veli Ozbolat, Effects of rear slant angles on the flow characteristics of Ahmed body, Experimental Thermal and Fluid Science, 2014, 57, 165-176.

[17] Katarzyna, S., Gabriel, W. and Derek, B.I., CFD modelling of air and oxy-coal combustion, International Journal of Numerical Methods for Heat \& Fluid Flow, 2014, 24( 4):825-844.

[18] https://en.wikipedia.org/wiki/Automobile_drag_coefficient

Ngày nhận bài: 15/06/2020

Ngày chấp nhận đăng: 30/03/2021 